\title{
PÉ DIABÉTICO: FATORES DE RISCO E IMPORTÂNCIA DO USO DE CALÇADOS APROPRIADOS COMO MEDIDA PREVENTIVA
}

\author{
Aline da Silva Santos ${ }^{1}$; Ana Mayra Andrade de Oliveira ${ }^{2}$; Gabriela Haana Maia dos \\ Santos Oliveira $^{3}$; Vitória Almeida Matos da Silva ${ }^{4}$; Lilia Paula de Souza Santos ${ }^{5}$ \\ 1.Bolsista PROBIC/UEFS. Graduanda em Medicina, Universidade Estadual de Feira de Santana. \\ Email: aliness1612@gmail.com \\ 2,Professora Doutora e Orientadora do Núcleo de Pesquisa de Endocrinologia, Departamento de Saúde, Universidade \\ Estadual de Feira de Santana. Email: anamayra@uol.com.br \\ 3. Bolsista PROBIC/UEFS. Graduanda em Medicina, Universidade Estadual de Feira de Santana. \\ Email: gabihaana@hotmail.com \\ 4. Bolsista PIBIC/FAPESB, Graduanda em Medicina, Universidade Estadual de Feira de Santana. \\ Email: vitoriauefs@gmail.com \\ 5. Mestre em Saúde Coletiva, Universidade Estadual de Feira de Santana. \\ Email: lipss13@hotmail.com.br
}

PALAVRAS-CHAVE: Diabetes Mellitus, Pé Diabético, Fator de Risco

\section{INTRODUÇÃO}

O Diabetes Mellitus (DM) é uma síndrome de etiologia múltipla, decorrente da secreção deficiente de insulina e/ou resistência à sua ação, isto é, da incapacidade da insulina de exercer seus efeitos adequadamente. Caracteriza-se por níveis de glicose plasmática elevados de forma crônica e distúrbios do metabolismo de carboidratos, lipídeos e proteínas (HALL, 2011).

O DM pode determinar o aparecimento de alterações sistêmicas do tipo microvascular, sendo uma das principais o Pé Diabético (PD) (CAIFA, 2011). O PD caracteriza-se pela presença de lesões nos pés decorrentes de neuropatias periféricas ( $90 \%$ dos casos), doença vascular periférica, dermatoses e deformidades, representando uma parcela significativa de internações hospitalares prolongadas, portanto condição deelevada morbidade e mortalidade. (SBD, 2016).

A educação do paciente, da família, profissionais de saúde como agentes comunitários de saúde sobre os cuidados gerais com os pés, corte adequado e cuidados com as unhas, cuidados para evitar infecções, uso de calçados adequados, higiene diária com cuidado especial ao secar a umidade entre os dedos, inspeção diária dos pés e dos sapatos, proibição do uso de calicidas e raspadores ou lâminas nos calos pelos pacientes, são medidas fundamentais para prevenção desta comorbidade do DM (CAIFA, 2011).

Observa-se que grande parte dos indivíduos que desenvolvem PD apresentam fatores de risco preveníveis e, a falta de conhecimento sobre os cuidados gerais com o pé representa fator decisivo para o aumento da sua prevalência e morbidade. Assim, estudos que visam identificar o grau de educação dos portadores de DM com relação aos cuidados com os seus pés são de extrema importância, para que estratégias sejam criadas com a finalidade de prevenção desta condição.

\section{METODOLOGIA}

Trata-se de estudo de corte transversal, quantitativo e do tipo exploratório. Nesta pesquisa, a amostra foi composta por indivíduos provenientes do ambulatório do Centro de Atendimento ao Diabético e Hipertenso (CADH). Foram incluídos todos os pacientes com diagnóstico de DM e PD de acordo comos critérios da Sociedade Brasileira de Diabetes (2016), apresentando mais de 10 anos de doença (classificados de acordo com o GTIPD Grupo de Trabalho Internacional sobre Pé Diabético, 2001). 
A coleta de dados foi realizada através da utilização de ficha contendo informações epidemiológicas e clínicas dos portadores de DM. Assim como, sobre o seu conhecimento a respeito dos cuidados com os seus pés, tipo de calçado comumente usado, sobre a disponibilidade dos serviços quanto à dispensação gratuita de órteses, calçados e próteses. Sapato adequado foi considerado como aquele que permite transpiração, oferece suporte às estruturas dos pés, tem bico que não aperta os dedos, é fechado e, se necessário, com palmilhas especiais (LIMA, I. G, 2017).

E, a respeito da avaliação de estratégias educacionais referentes ao cuidado com o PD realizadas na Unidade de Saúde. Ao final foi realizada uma reunião na Unidade de Saúde, com os pacientes que participaram da pesquisa, em que foram abordados assuntos relacionados às medidas de cuidado integral com os pés enfatizando sua importância na prevenção de possíveis complicações.

O método de análise dos dados utilizado foi o de Análise de Conteúdo. De acordo com os aspectos éticos o estudo foi conduzido em conformidade com o Comitê de Ética e Pesquisa (CEP) e todos os pacientes assinarão o Termo de Consentimento Livre e Esclarecido (TCLE). Os pacientes possuíram autonomia sobre a participação de qualquer etapa da pesquisa. As informações obtidas foram analisadas em conjunto com as de outros pacientes, e não será divulgada a identificação de nenhum paciente. O estudo ao qual o projeto se vincula foi aprovado pelo CONSEPE local (049/2013).

\section{RESULTADOS}

O estudo contou com a participação de 113 indivíduos com idade média de 65,54 \pm 8,92 anos. Sendo 75,9\% da amostra composta por mulheres. Com relação a etnia, declararamse brancos, negros e pardos, 8,6\%, 50,9\% e 37,9\%, respectivamente. A renda mensal correspondeu a 1 a 3 salários para 81\% dos indivíduos. Quanto ao grau de escolaridade, 27\% declararam-se analfabetos e $60 \%$ afirmaram ter estudado apenas até o ensino fundamental. $75 \%$ da amostra era composta por indivíduos com duração média de doença de 65,58 anos e $64,7 \%$ eram sedentários.

Com relação às comorbidades associadas ao DM, a hipertensão arterial sistêmica (HAS) (88,5\%) teve lugar de destaque, seguida da dislipidemia (60,2\%). Quanto ao uso de sapato adequado, 82 (71,9\%) dos participantes apresentavam calçados inadequados, 20\% faziam uso de sapatos com solado rígido, ou seja, a maioria dos indivíduos utiliza calçados que não protegem o suficiente contra lesões (Tabela 3).

\section{Tabela 3 - Análise do tipo de calçado utilizado pela população estudada}

\begin{tabular}{lcc}
\hline Variáveis & $\mathbf{n}$ & $\mathbf{\%}$ \\
\hline Solado do Sapato & & \\
$\quad$ Rígido & 23 & 20,2 \\
$\quad$ Flexível & 90 & 78,9 \\
Deformidade provocada pelo sapato & & \\
$\quad$ Não & 104 & 91,2 \\
$\quad$ Sim & 9 & 7,9 \\
Calçado Adequado & & \\
$\quad$ Não & 81 & 71,9 \\
$\quad$ Sim & 31 & 28,1 \\
\hline
\end{tabular}


Entre os entrevistados 51\% consideram o uso de calçados adequados importante para prevenir possíveis complicações e $20 \%$ afirmam que eles ajudam a melhorar a caminhada. Com relação ao conhecimento dos participantes do estudo acerca dos cuidados com os pés, ao acesso e ao conhecimento a respeito da distribuição gratuita de órteses, sapatos e próteses para portadores de DM, foi evidenciado um grau significativo de déficit (Tabela 4).

Tabela 4 - Análise do conhecimento da síndrome do pé diabético e dos cuidados necessários.

\begin{tabular}{lcc}
\hline Variáveis & $\mathbf{N}$ & $\mathbf{\%}$ \\
\hline Orientação sobre o DM na USF & & \\
$\quad$ Não & 5 & 4,4 \\
Sim & 108 & 94,7 \\
Conhece o sistema de distribuição & & \\
gratuita de órteses e calçados & & \\
$\quad$ Não & 100 & 87,7 \\
Sim & 13 & 11,4 \\
Necessária distribuição gratuita de & & \\
sapatos & & \\
$\quad$ Não & 3 & 2,6 \\
Sim & 110 & 96,5 \\
\hline
\end{tabular}

Entende-se que o acesso precário à rede de saúde, a insuficiência de treinamento de profissionais em relação a $\mathrm{PD}$, a fragilidade na regulação de referência e contra referência, bem como registros e monitorização de amputações por DM ineficientes podem agravar a problemática (ADA, 2017). Esses dados enfatizam a necessidade da implementação de estratégias educacionais e assistenciais acessíveis que facilitem o entendimento e o apoio a essa população, objetivando simplificar a compreensão do autocuidado para uma maior abrangência dos pacientes, independentemente de sua escolaridade.

\section{CONCLUSÃO}

Esse estudo evidenciou um déficit em ações especializadas, direcionadas para prevenção e tratamento do PD, uma complicação de elevada morbidade e custo social e econômico para população.

Diante da complexidade do tratamento desta complicação entende-se como fundamental uma maior interação entre equipe multiprofissional e paciente, com objetivo preventivo, terapêutico e de reabilitação

\section{REFERÊNCIAS}

HALL, J. E. Tratado de Fisiologia Médica. 12 ed.-Rio de Janeiro; 2011.

CAIAFA, J. S. et al. Atenção integral ao portador de pé diabético. J. vasc. bras. [online], v.10, n.4, p. 1-32, 2011.

Sociedade Brasileira de Endocrinologia e Metabologia, Diabetes. Disponível em $<$ http $/ /$ www.endocrino.org.br $>$ Acesso em Abril de 2016.

Grupo de trabalho internacional sobre pé diabético. Consenso internacional sobre pé diabético. Brasília Secretaria de Estado de Saúde do Distrito Federal; 2001.

LIMA, I. G. et al. Educar para prevenir: a importância da informação no cuidado do pé diabético . R. Conexão UEPG, v. 10, n. 1, p 186-194, 2017 
American Diabetes Association.Standards of Medical Care in Diabetes. Diab Care. 2017, Jan;37(Suppl 1). 